\title{
可见光催化促进的金属有机基元反应
}

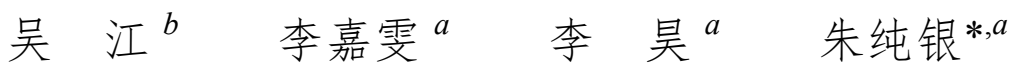 \\ ( ${ }^{a}$ 江苏大学化学化工学院 镇江 212013$)$ \\ $\left({ }^{b}\right.$ 中国科学院上海有机化学研究所 金属有机化学国家重点实验室 上海 200032)
}

\begin{abstract}
摘要 绝大多数金属有机反应都涉及到氧化加成、还原消除、转金属化等基元反应. 一般认为, 这些基元反应都是通 过电子成对转移来实现的，而这一方式有时存在较高能垒，导致反应较难发生. 近年来，可见光催化已经发展成为一 种重要的活化方式，可以通过温和的方式高选择性地诱导氧化还原反应. 基于此, 可见光催化促进的金属有机基元反 应可以产生一些新的反应活性, 从而越来越受到学界的关注. 按照基元反应的类型综述了这一领域的最新进展.

关键词 金属有机反应; 基元反应; 可见光催化
\end{abstract}

\section{Visible Light Photoredox Catalysis Mediated Elementary Steps in Organometallic Reactions}

\author{
$\mathrm{Wu}_{\text {, Jiang }}{ }^{b} \quad \mathrm{Li}_{\text {, Jiawen }}{ }^{a} \quad \mathrm{Li}_{\text {, Hao }}{ }^{a} \quad$ Zhu, Chunyin*,a \\ $\left({ }^{a}\right.$ School of Chemistry and Chemical Engineering, Jiangsu University, Zhenjiang 212013) \\ $\left({ }^{b}\right.$ State Key Laboratory of Organometallic Chemistry, Chinese Academy of Sciences, Shanghai 200032)
}

\begin{abstract}
Many organometallic reactions involve three elementary steps including oxidative addition, transamination, and reductive elimination. But sometimes very high barrier exists for this approach, leading to the failure of the reactions. The key development in recent reports is the implementation of visible-light photocatalysts as a means to induce the desired redox processes in a mild and selective manner. In this context, organometallic reactions mediated by photoredox catalysts could give rise to novel reactivity, thus this area has drawn much attention in organic chemistry community. In this review, prominent examples from the recent literatures are organized on the basis of the elementary transformation enabled by photoredox catalysis.

Keywords organometallic reactions; elementary steps; visible light photoredox
\end{abstract}

金属有机反应是现代合成化学的重要基石之一, 可 高效地构建各种碳碳键和碳杂键, 已被广泛地应用于药 物、天然产物等复杂化合物的合成中 ${ }^{[1]}$. 大部分金属有 机反应通常包含氧化加成和还原消除等基元反应，而这 些基元反应一般会涉及到金属价态的改变. 一直以来, 人们较多地依赖于金属和配体自身的特性来调控金属 变价, 实现氧化加成和还原消除等基元反应. 可见光催 化方法学是近年来发展起来的一种强大的合成手段, 以 其低能耗、条件温和、无污染等特点而广受关注, 给有 机合成化学带来了新的机遇 ${ }^{[2]}$. 这一催化模式具有高活 性和氧化还原特性, 可以通过单电子转移的机制实现金 属的氧化还原，从而促进金属有机基元反应，特别是那
些仅依赖金属和配体自身能力而无法实现的基元反应. 近年来，很多文献报道了可见光催化促进的氧化加成、 还原消除以及转金属化反应，因此，本文综述了这些反 应的最新进展及其反应机理的探讨.

\section{1 可见光催化促进的氧化加成}

氧化加成是亲电组分与金属中心的成键作用，同时 伴随着金属中心形式上的双电子氧化, 大多数反应表现 为碳卤键或类碳卤键对金属的一步协同的氧化加成. 但 是，很多金属和亲电试剂经常无法通过这一协同的反应 机理进行反应. 而很多研究表明烷基自由基可以方便地 加成到金属中心 ${ }^{[3]}$, 例如: 光催化产生的异丙基自由基

\footnotetext{
* Corresponding author. E-mail: zhucycn@gmail.com

Received April 18, 2017; revised May 22, 2017; published online June 7, 2017.

Project supported by the Qing Lan Project of Jiangsu Province.

江苏省 “青蓝工程” 中青年学术带头人培养对象资助项目.
} 
可以加速异丙基碘对 $\operatorname{Pt}(\mathrm{II})$ 配合物的氧化加成 ${ }^{[4]}$; 类似 地, 氟代烷基碘也是通过链式的自由基机理对 $\mathrm{Au}(\mathrm{I})$ 配 合物进行氧化加成 ${ }^{[5]}$. 基于此, 人们开始考虑利用光催 化产生自由基促进氧化加成, 从而实现催化的有机合 成. 这方面最早的例子是 2011 年 Sanford 等 $^{[6]}$ 报道的钯 催化的导向 $\mathrm{C}-\mathrm{H}$ 键活化. 他们利用芳基重氮盐在可见 光催化下, 室温即可产生高价钯中间体, 无需高温加热 的剧烈条件. 所得 Pd(IV)中间体 1-C 发生还原消除可以 得到各种取代的芳基化产物. 此法适用于不同的定位基 团和芳基重氮盐，以及二芳基碘盐(Scheme 1).

随后这一策略被利用到了金催化的反应中, 人们通 过芳基重氮盐或者二芳基碘盐可获得高活性的三价金 中间体, 从而利用该中间体在温和的条件下实现了一系 列的重要有机转化. 例如, Glorius 等 ${ }^{[7]}$ 在 2013 年报道了 利用金和光催化的组合催化策略实现的烯烃的双功能 化反应. 该反应适用于不同取代的含氧/氮烯烃和芳基 重氮盐, 在室温和家用荧光灯的照射下, 可以合成各类 芳基杂环化合物，产率中等到良好(Eq. 1).

作者认为该反应的第一步是被金配位的双键 4 被分 子内的氧或者氮亲核试剂进攻, 从而生成一价烷基金中 间体 4-A. 接着，该一价金中间体首先与芳基自由基加 成为二价金中间体 4-B, 随后被光催化剂单电子氧化生
成三价金中间体 4-C. 最后通过还原消除得到目标产物, 同时再生一价金催化剂(Scheme 2).

利用类似策略，该课题组 ${ }^{[8]}$ 还报道了分子间烯烃的 醚化芳基化反应. 该反应适用于不同取代的末端烯烃和 芳基重氮盐或者二芳基碘盐，在室温和家用苂光灯的照 射下，可以合成各类芳基杂环化合物，个别底物产率可 达优秀(Scheme 3).

类似地, 最近 Ollivier 和 Fensterbank 等 ${ }^{[9}$ 报道了利 用炔烃的醚化烷基化合成苯并呋喃的反应，该反应以各 种取代的炔基苯酚和芳基重氮盐为底物，条件温和，产 率良好到优秀. 作者认为该反应也经历了光催化氧化产 生三价金中间体的历程(Eq. 2).

可见光促进的氧化加成反应还被应用在一系列金 催化的芳基化重排反应. 例如, 2014 年 Toste 小组 ${ }^{[10]}$ 报 道了利用金和光催化的组合策略实现的氧化芳基化扩 环反应。该反应以不同的烯基或联烯基环醇，和重氮盐 为底物，在金和光催化作用下，可以制得各种官能团化 的环酮(Eq. 3).

通过包括时间分辨 FT-IR 在内的各种机理实验的考 察，作者认为该反应首先通过光催化将芳基重氮盐还原 为芳基自由基，而该自由基可与一价金催化剂反应生成 二价金中间体. 接着，二价金中间体又经光催化氧化为

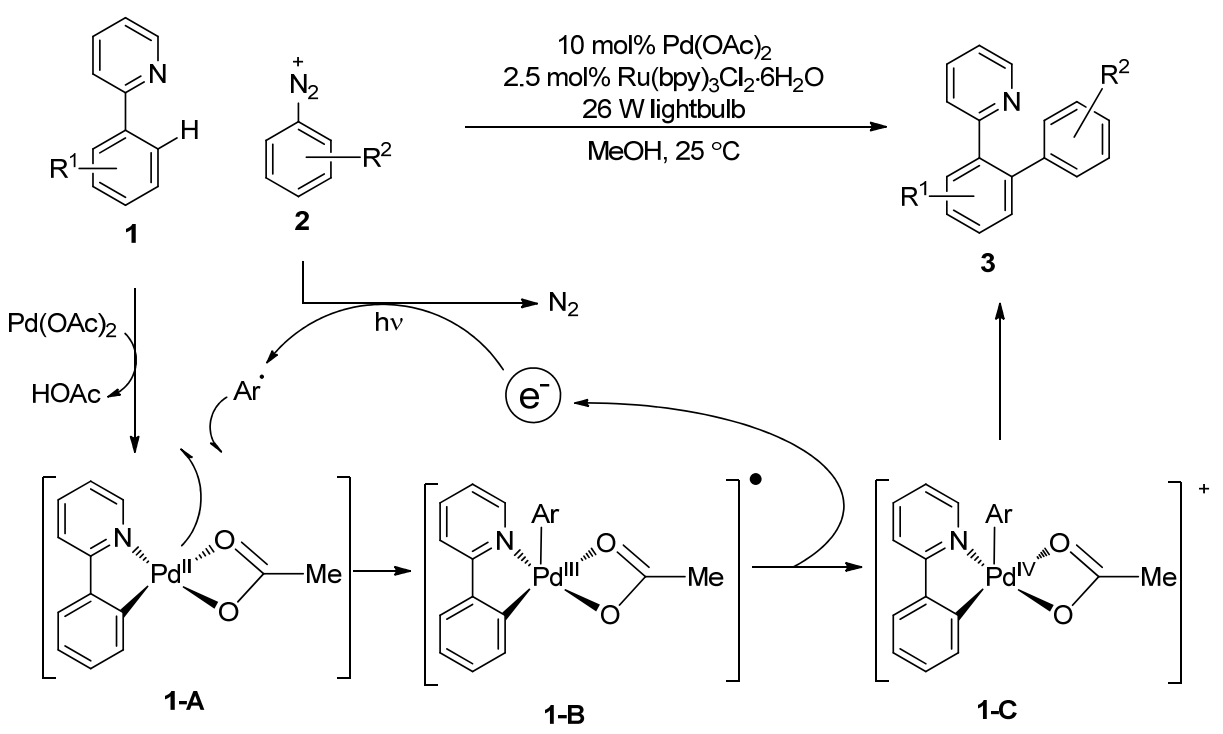

图 1 经由光催化产生钯(IV)中间体的钯催化的 $\mathrm{C}-\mathrm{H}$ 键官能团化反应

Scheme 1 Palladium-catalyzed C-H functionalization via $\mathrm{Pd}(\mathrm{IV})$ intermediates generated by photoredox catalysis

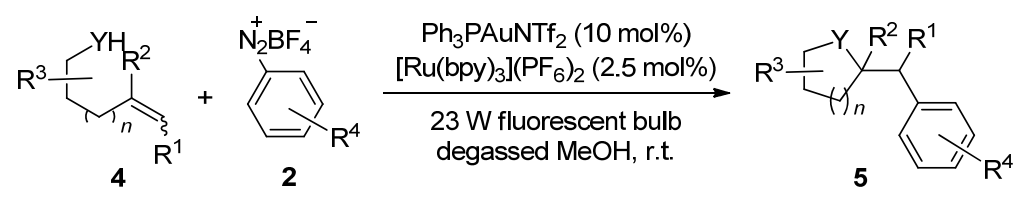




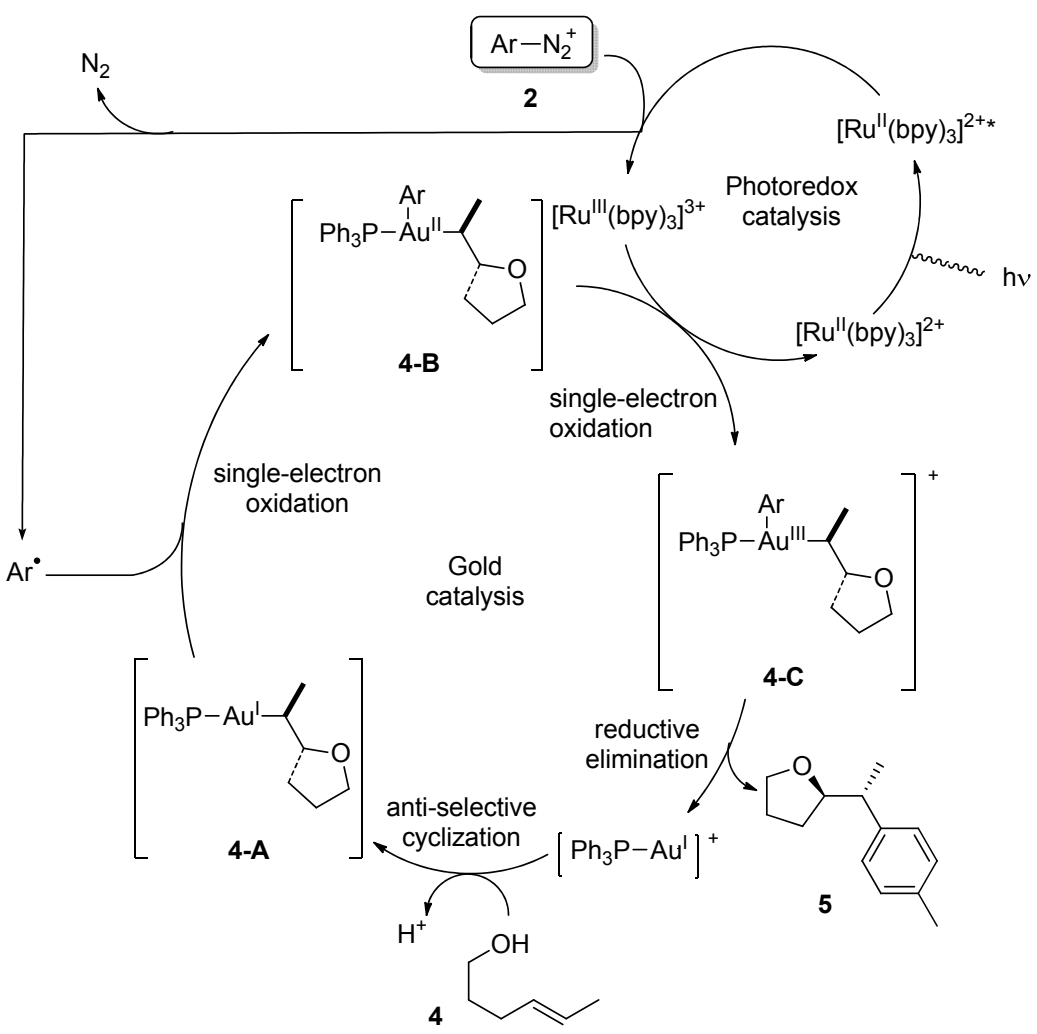

图 2 金和光催化剂共同催化的含氧/氮烯烃与芳基重氮盐的反应机理

Scheme 2 Proposed mechanism of the dual visible light photoredox and gold-catalyzed oxy- and aminoarylation of alkenes with aryldiazonium salts

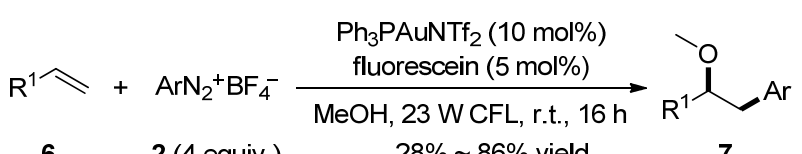

$6 \quad 2$ (4 equiv.)

$28 \% \sim 86 \%$ yield
$\mathrm{Ph}_{3} \mathrm{PAuNTf}_{2}$ (10 mol\%)

$\mathrm{R}^{1} \curvearrowright+\left[\mathrm{Ar}_{2} \mathrm{I}^{+} \mathrm{BF}_{4}^{-} \underset{\mathrm{R}^{3} \mathrm{OH} \text {, blue LEDs, r.t., } 20 \mathrm{~h}}{\stackrel{\left[\operatorname{lr}(\mathrm{ppy})_{2}(\mathrm{dtbbpy})\right] \mathrm{PF}_{6}(5 \mathrm{~mol} \%)}{\longrightarrow}}\right.$

$6 \quad 8$ (4 equiv.)

$26 \% \sim 91 \%$ yield

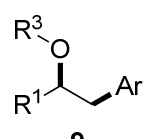

图 3 金和光催化剂共同催化的烯烃的醚化芳基化反应

Scheme 3 Dual visible light photoredox and gold-catalyzed oxyarylation of alkenes<smiles>[R]c1cccc(O)c1C#Cc1ccccc1</smiles><smiles>[R][X]1ccccc1[N+]</smiles>

$\mathrm{Ph}_{3} \mathrm{PAuCl}(10 \mathrm{~mol} \%)$

$\underset{\mathrm{MeOH}}{\stackrel{\left[\mathrm{Ru}(\mathrm{bpy})_{3}\right]\left(\mathrm{PF}_{6}\right)_{2}(2.5 \mathrm{~mol} \%)}{\longrightarrow}}$

23 W fluorescent bulb up to $86 \%$ yield

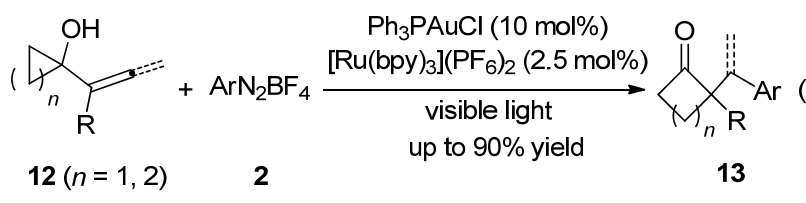

三价金中间体，同时再生光催化剂. 最后，三价金中间 体与 $\pi$ 键配位, 导致开环和随后的还原消除而生成最终 产物(Scheme 4).

炔丙醇也可在此策略下发生芳基化重排反应. 2016 年, Shin 等 ${ }^{[11]}$ 报道了利用炔丙醇和芳基重氮盐合成 $\alpha$-芳 基酮的反应. 以 $\mathrm{Ph}_{3} \mathrm{PAuCl}$ 和 $\left[\mathrm{Ru}(\mathrm{bpy})_{3}\right](\mathrm{PF} 6)_{2}$ 为催化剂, 在蓝光 LED 灯的照射下，该反应产率可达 $95 \%$ (Eq. 4).

作者认为该反应首先通过光催化利用芳基重氮盐 将一价金氧化为三价金配合物. 然后，在氢键的介导下， 甲醇进攻被三价金配位的炔烃而生成烯基金中间体 14-B. 该烯基金中间体再发生消除反应得到联烯基金中 间体 14-C, 进而发生异构和水解生成最终产物 (Scheme 5).

在 Shin 等的工作报道后不久, Glorius ${ }^{[12]}$ 和 Alcaide 等 ${ }^{[13]}$ 也报道了他们利用光和金催化剂共催化炔丙醇和 芳基重氮盐的串联重排/偶联反应。他们利用易于制备 


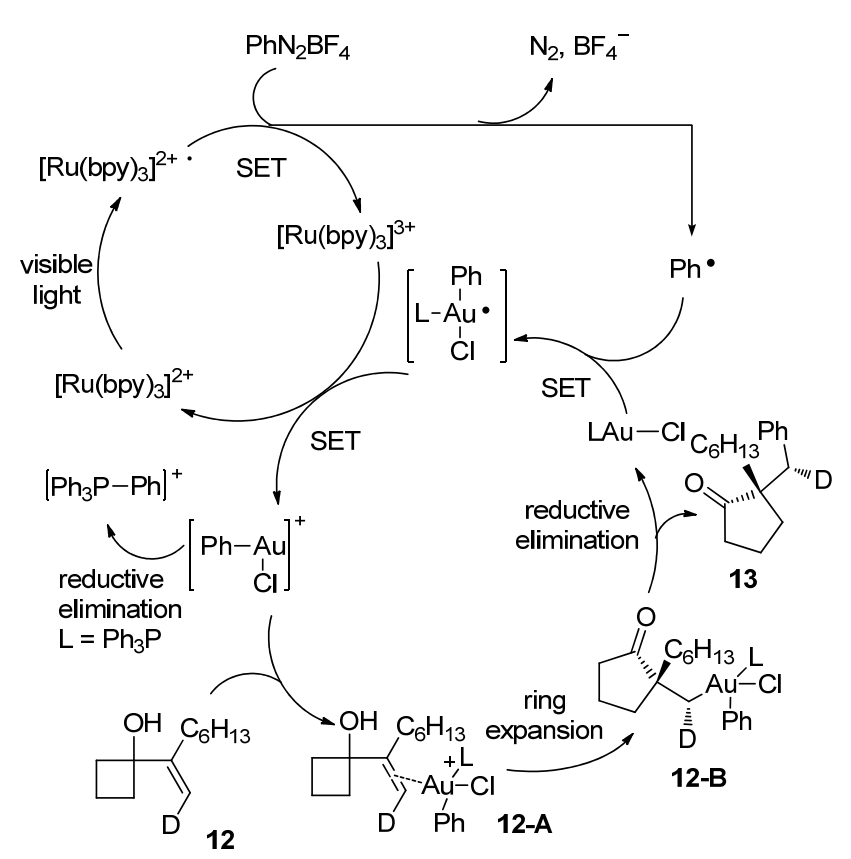

图 4 光催化促进的、金催化氧化芳基化扩环反应机理

Scheme 4 Proposed mechanism of the photoredox-promoted, gold-catalyzed ring expansion-arylation reaction

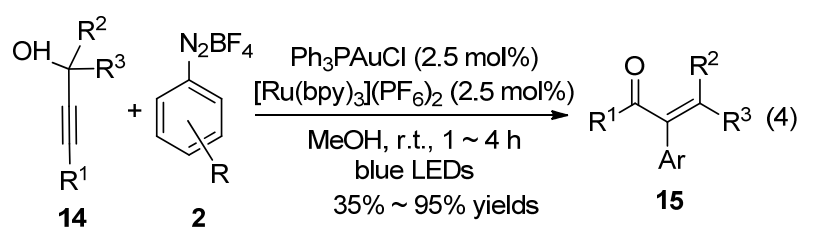<smiles></smiles>

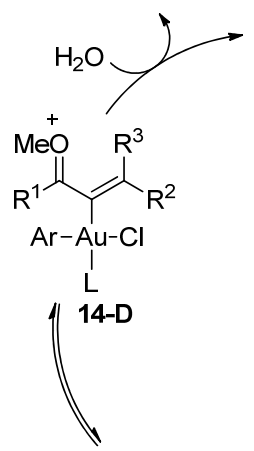<smiles>[GeH3]</smiles>

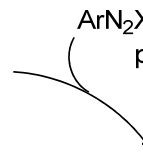
photoredox<smiles>[R1]C#CC([R])([R])O</smiles><smiles>[R]CC([R])([Al])C([R])([Y19])COC</smiles>

图 5 利用炔丙醇和芳基重氮盐合成 $\alpha$-芳基酮的反应机理 Scheme 5 Proposed mechanism of the reaction of propargyl alcohols with aryl diazonium salts

的炔丙醇作为底物通过 Meyer-Schuster 重排反应合成 $\alpha$ 芳基烯酮; 而炔的水合反应也可以与交叉偶联反应串联
选择性地合成 $\alpha$-芳基酮. 两个反应均是在室温、可见光 照射的条件下进行，可见光来源于易于获得的光源、甚 至是日光(Scheme 6).
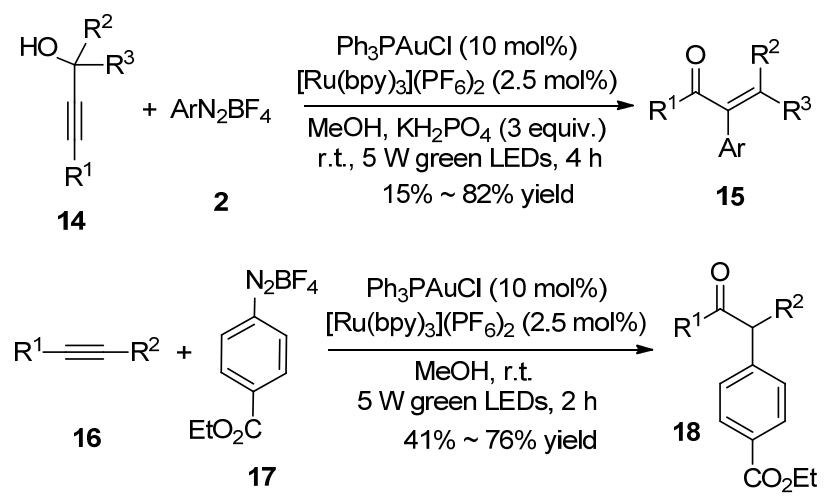

Scheme 6 金/光共催化的炔双功能化

Scheme 6 Alkyne difunctionalization by dual gold/photoredox catalysis

利用类似的光和金催化剂共同催化的策略, Alcaide 等 ${ }^{[13]}$ 研究了含有脂肪族、芳香族取代基的伯/仲炔丙醇 与芳基重氮盐的 Meyer-Schuster/芳基化反应，产率中等 到良好(Eq. 5).
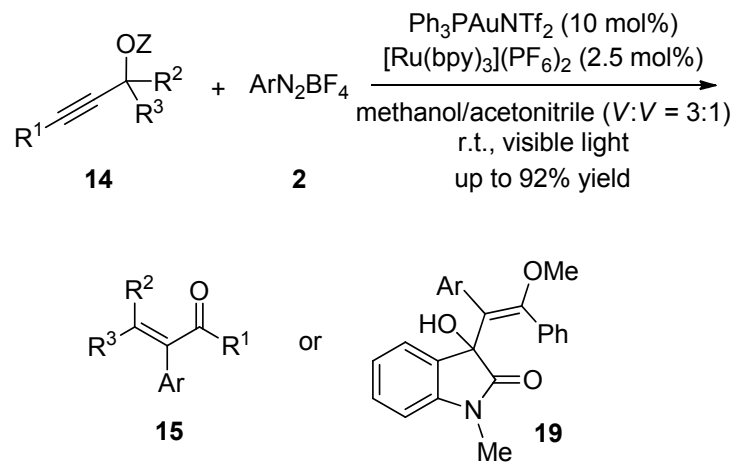

另外，可见光促进的氧化加成反应还被应用在一系 列金催化的交叉偶联反应中. 2015 年, Toste 小组 ${ }^{[14]}$ 报道 了金和光催化剂共催化的 $\mathrm{C}-\mathrm{P}$ 交叉偶联反应. 在 $\mathrm{Ph}_{3} \mathrm{PAuCl}(10 \mathrm{~mol} \%)$ 和 $\left[\mathrm{Ru}(\mathrm{bpy})_{3}\right]\left(\mathrm{PF}_{6}\right)_{2}(2 \mathrm{~mol} \%)$ 的存在 下，芳基重氮盐可以与不同类型的 $\mathrm{P}(\mathrm{O}) \mathrm{H}$ 化合物反应得 到偶联的芳基膦酸酯/亚膦酸酯，收率可达到 $90 \%$. 值得 一提的是，如果将金催化剂替换为钯、银或铜盐，产物 的收率很低、甚至没有产物产生，这些结果表明，金催 化在这类反应中的独特性(Eq. 6).

包括 $\mathrm{C}\left(\mathrm{sp}^{2}\right)-\mathrm{C}\left(\mathrm{sp}^{2}\right)$ 和 $\mathrm{C}(\mathrm{sp})-\mathrm{C}\left(\mathrm{sp}^{2}\right)$ 在内的 $\mathrm{C}-\mathrm{C}$ 的 交叉偶联反应也有报道. 这些反应以芳基重氮盐作为自 由基源在光催化的条件下将 $\mathrm{Au}(\mathrm{I})$ 氧化为活性的 $\mathrm{Au}(\mathrm{III})$, 另一偶联的组分可以是炔或芳基硼酸. 2016 年 Glorius 小组 ${ }^{[15]}$ 报道了一个光和金催化剂共催化的端基炔芳基 

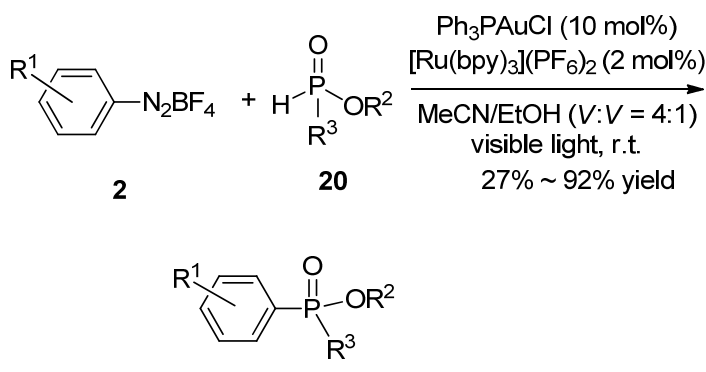

(6)

21

化反应. 在 $10 \mathrm{~mol} \%\left((p-\mathrm{MeO}) \mathrm{C}_{6} \mathrm{H}_{4}\right)_{3} \mathrm{PAuCl}$ 和 $0.5 \mathrm{~mol} \%$ $\left[\mathrm{Ru}(\mathrm{bpy})_{3}\right]\left(\mathrm{PF}_{6}\right)_{2}$ 的条件下, 不同的烃基/芳基端炔与不 同的芳基重氮盐反应，得到偶联产物，产率可达 79\% (Scheme 7).
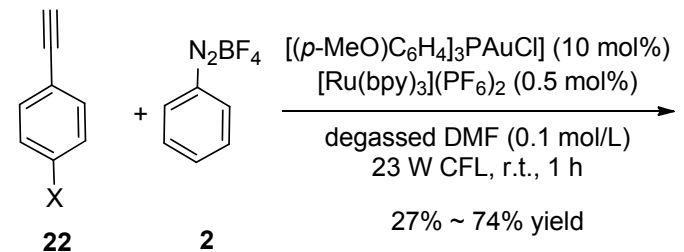

$\mathrm{X}=\mathrm{CN}, \mathrm{CH}_{3} \mathrm{O}$

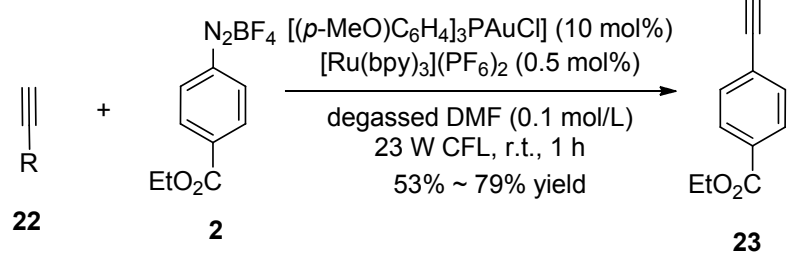

Scheme 7 金/光共催化的端基炔和芳基重氮盐的 $\mathrm{C}(\mathrm{sp})-\mathrm{H}$ 芳基化反应

Scheme 7 Dual gold/photoredox-catalyzed C(sp) - H arylation of terminal alkynes with diazonium salts

该反应的可能机理是, 首先, 光激发 $\left[R u(b p y)_{3}\right]^{2+}$ 为 激发态的* $\left[\mathrm{Ru}(\mathrm{bpy})_{3}\right]^{2+}$, 然后与芳基重氮盐经历单电子 转移(SET)产生芳基自由基和氧化态的 $\left[\mathrm{Ru}(\mathrm{bpy})_{3}\right]^{3+}$, 芳基自由基与 $\mathrm{Au}(\mathrm{I})$ 催化剂反应产生 $\mathrm{Au}(\mathrm{II})$. 其次, $\mathrm{Au}(\mathrm{II})$ 再和另芳基重氮盐发生单电子转移反应产生亲电阳离 子 $\mathrm{Au}(\mathrm{III})$ 中间体, 然后 $\mathrm{Au}(\mathrm{III})$ 中间体与炔配位, 得到炔 $-\mathrm{Au}(\mathrm{III})$ 配合物. 最后, 炔- $\mathrm{Au}(\mathrm{III})$ 配合物经还原消除反 应再生 $\mathrm{Au}(\mathrm{I})$ 催化剂和交叉偶联的产物(Scheme 8).

除了端基炔，炔基三甲基硅烷也可以用作 $\mathrm{C}(\mathrm{sp})$ $\mathrm{C}\left(\mathrm{sp}^{2}\right)$ 交叉偶联反应的底物. 2016 年, Toste 等 ${ }^{[16]}$ 报道了 $\mathrm{Ar}_{3} \mathrm{PAuCl}$ 和 $\mathrm{Ru}(\mathrm{bpy})_{3}(\mathrm{PF} 6)_{2}$ 共催化的炔基三甲基硅烷 和芳基重氮盐的偶联反应. 该反应条件温和, 产率可以 达到 $82 \%$, 并具有好的官能团耐受性(Eq. 7).

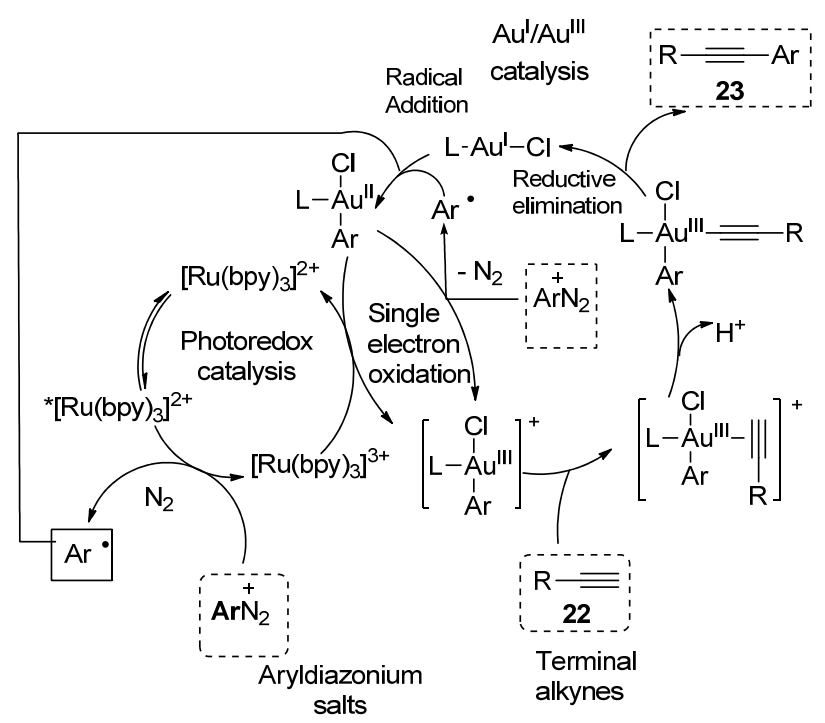

Scheme 8 金和光催化剂共催化的端基炔和重氮盐的 C(sp)$\mathrm{H}$ 芳基化的可能的反应机理

Scheme 8 Proposed mechanism for the dual gold/photoredoxcatalyzed $\mathrm{C}(\mathrm{sp})-\mathrm{H}$ arylation of terminal alkynes with diazonium salts

$$
\text { 24 }
$$

金/光催化剂共同催化的 $\mathrm{C}\left(\mathrm{sp}^{2}\right)-\mathrm{C}\left(\mathrm{sp}^{2}\right)$ 的交叉偶联 反应通常是以芳基重氮盐和芳基嗍酸作为底物的. 2016 年, Hermange/Fouquet 和 Lee 两个研究小组分别独立报 道了他们在该领域的研究工作. Hermange 和 Fouque 研 究小组 ${ }^{[17]}$ 发展了一种合成二芳基化合物的方法. 该反 应是在蓝光 LED 灯照射下，以芳基重氮盐和芳基硼酸 为反应物, $\mathrm{PPh}_{3} \mathrm{AuCl}$ 作催化剂, $\mathrm{CsF}$ 作碱, 乙腈作溶剂, 9-mesityl-10-acridinium tetrafluoroborate 或者 $\mathrm{Ru}(\mathrm{bpy})_{3}$ $\left(\mathrm{PF}_{6}\right)_{2}$ 作为光敏剂. 该反应也适用于带有多种官能团的 底物，如澳代芳基、碘代芳基、醛和醇(Eq. 8). 通过化学 计量的 $\mathrm{PPh}_{3} \mathrm{AuPh}$ 的 ${ }^{31} \mathrm{P}$ 和 ${ }^{1} \mathrm{H} \mathrm{NMR}$ 谱图, 作者研究了 该反应的机理。研究表明，在 9-mesityl-10-acridinium tetrafluoroborate 存在下, 蓝光 LED 灯照射, $\mathrm{PPh}_{3} \mathrm{AuPh}$ 和 芳基重氮盐反应得到目标的偶联产物.

根据这些研究结果和以前的关于金/光催化剂共催 化的文献报道，该反应的机理可能是：首先, $\mathrm{PPh}_{3} \mathrm{AuCl}$ 和芳基硼酸发生转金属化产生 $\mathrm{PPh}_{3} \mathrm{Au}^{\mathrm{I}} \mathrm{Ar}^{\prime}$, 从而触发了 反应. 其次，与芳基自由基发生氧化加成反应，生成氧 化态的 $\mathrm{Au}(\mathrm{II})$ 中间体 26-B, $\mathrm{Au}(\mathrm{II})$ 中间体再发生单电子氧 


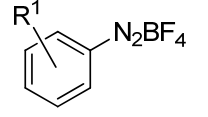

2

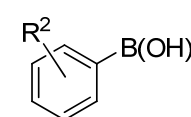

26

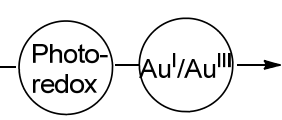

$47 \% \sim 92 \%$ yield<smiles>[R]c1ccc(-c2ccc([R])cc2)cc1</smiles>

化反应生成 $\mathrm{Au}(\mathrm{III})$ 中间体 26-C. 最后, $\mathrm{Au}(\mathrm{III})$ 配合物经 过还原消除反应生成二芳基化合物, 并再生 $\mathrm{Au}(\mathrm{I})$ 催化 剂(Scheme 9).

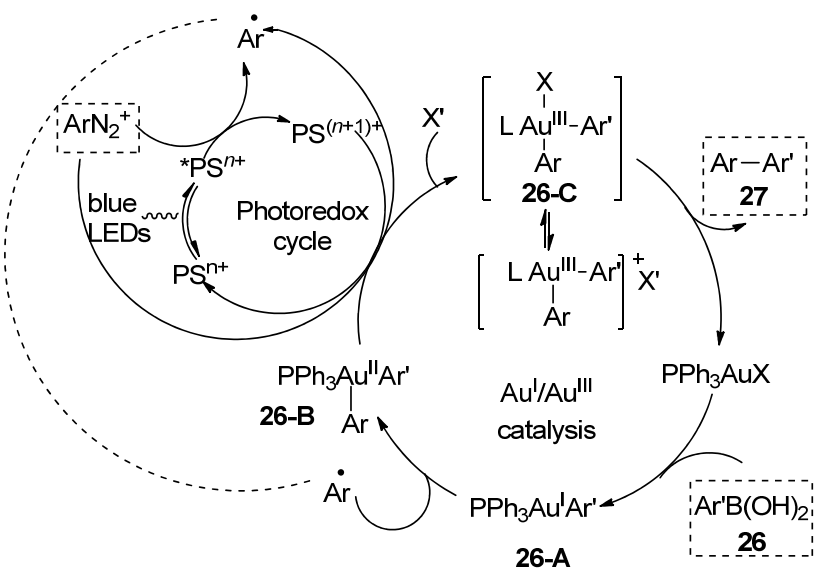

Scheme 9 芳基硼酸与芳基重氮盐偶联反应的可能机理 Scheme 9 Proposed mechanism for the couplings of arylboronic acids with aryldiazonium salts

几乎与此同时, Lee 等 ${ }^{[18]}$ 也报道了芳基硼酸和芳基 重氮盐的温和的、氧化的、非碱性的交叉偶联反应. 在 $5 \mathrm{~mol} \% \mathrm{PPh}_{3} \mathrm{AuNTf}_{2}$ 和 $2.5 \mathrm{~mol} \%\left[\mathrm{Ru}(\mathrm{bpy})_{3}\right]\left(\mathrm{PF}_{6}\right)_{2}$ 存在 下，各种芳基嗍酸可以顺利地与不同的芳基重氮盐反应 生成目标的偶联产物，收率可达到 92\% (Eq. 9).

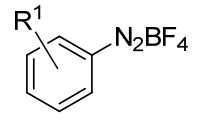

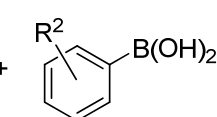

26

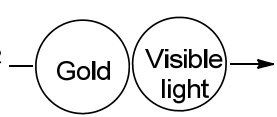

up to $92 \%$ yield<smiles>[R]c1ccc(-c2ccccc2)cc1</smiles>

通过对 ${ }^{31}$ P NMR 的谱图分析，对该反应的机理进行 了详细的研究，并给出了两个可能的反应机理.一种可 能的机理是: 芳基硼酸和 $\mathrm{Au}(\mathrm{I})$ 的转金属化发生在 $\mathrm{Au}(\mathrm{I})$ 氧化为 $\mathrm{Au}(\mathrm{III})$ 之前; 另外一个可能的机理是: $\mathrm{Au}(\mathrm{I})$ 氧化 为 $\mathrm{Au}(\mathrm{III})$ 先于 $\mathrm{Au}(\mathrm{I})$ 的转移金属化(Scheme 19).

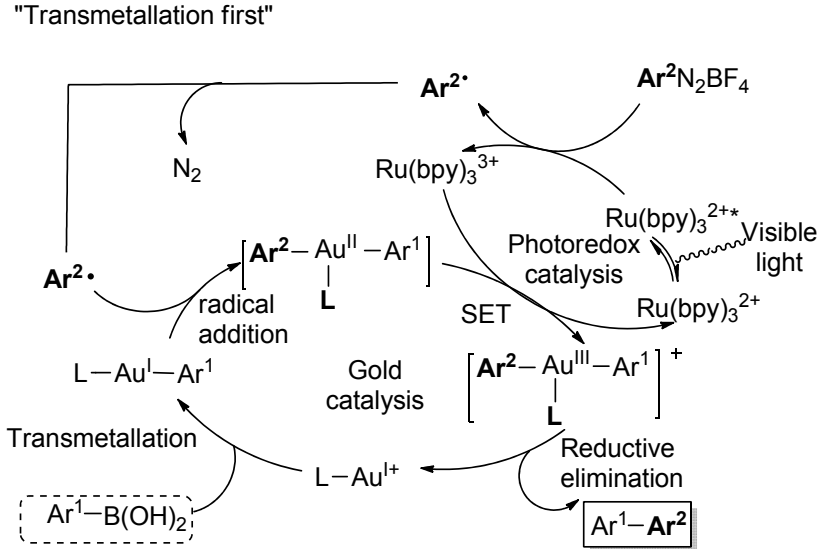

"Oxidation first"

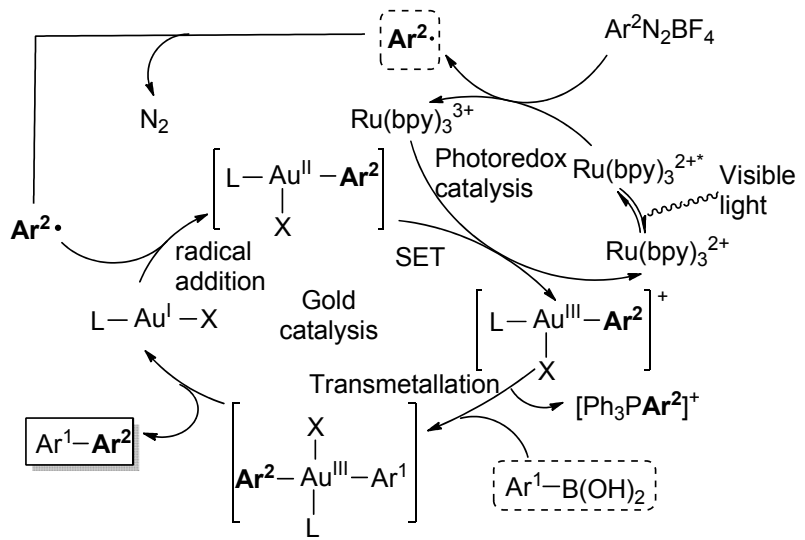

Scheme 10 金和光催化剂共催化的 $\mathrm{C}\left(\mathrm{sp}^{2}\right)-\mathrm{C}\left(\mathrm{sp}^{2}\right)$ 交叉偶联 反应的两个可能机理

Scheme 10 Two distinct pathways for the dual gold photoredox $\mathrm{C}\left(\mathrm{sp}^{2}\right)-\mathrm{C}\left(\mathrm{sp}^{2}\right)$ cross coupling

\section{2 可见光催化促进的还原消除}

还原消除一般是金属配体之间的成键，同时伴随着 金属中心形式上的双电子还原. 作为偶联反应的关键步 骤，还原消除被广泛研究，大量的金属和配体被应用于 不同的反应，但是仍然有很多还原消除很慢，甚至很难 发生的情况. 光催化的介入也可以促进还原消除反应. 2014 年 Doyle \& MacMillan 等 ${ }^{[19]}$ 报道了镍和光催化剂协 同催化的 $\mathrm{C}\left(\mathrm{sp}^{3}\right)-\mathrm{C}\left(\mathrm{sp}^{2}\right)$ 偶联反应，成功实现了一系列 烷基羧酸与芳基卤化物的脱羧偶联(Eq. 10).

对于反应的机理，作者认为首先芳基卤化物与 $\mathrm{Ni}(0)$ 发生氧化加成产生 $\mathrm{Ni}(\mathrm{II})$ 中间体 29-A, 同时, 羧酸 在光催化条件下脱羧产生烷基自由基，然后该自由基加 成到 $\mathrm{Ni}(\mathrm{II})$ 中间体上生成 $\mathrm{Ni}$ (III)中间体 29-B. 接着, $\mathrm{Ni}(\mathrm{III})$ 中间体发生还原消除生成 $\mathrm{Ni}(\mathrm{I})$ 中间体，最后 $\mathrm{Ni}(\mathrm{I})$ 被处于低价态的光敏剂还原为 $\mathrm{Ni}(0)$. 整个过程通过单 电子转移实现了 $\mathrm{Ni}(0) \rightarrow \mathrm{Ni}(\mathrm{II}) \rightarrow \mathrm{Ni}(\mathrm{III}) \rightarrow \mathrm{Ni}(\mathrm{I})$ 的循环, 

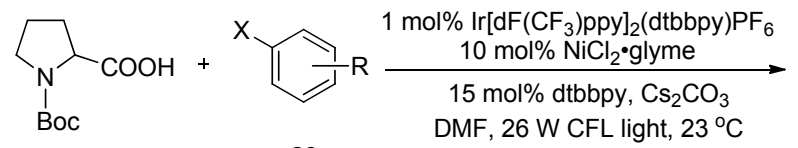

29

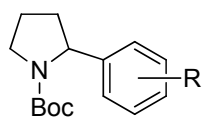

30

克服了传统 $\mathrm{Ni}(0) \rightarrow \mathrm{Ni}(\mathrm{II}) \rightarrow \mathrm{Ni}(0)$ 机制中还原消除较困难 的问题(Scheme 11).

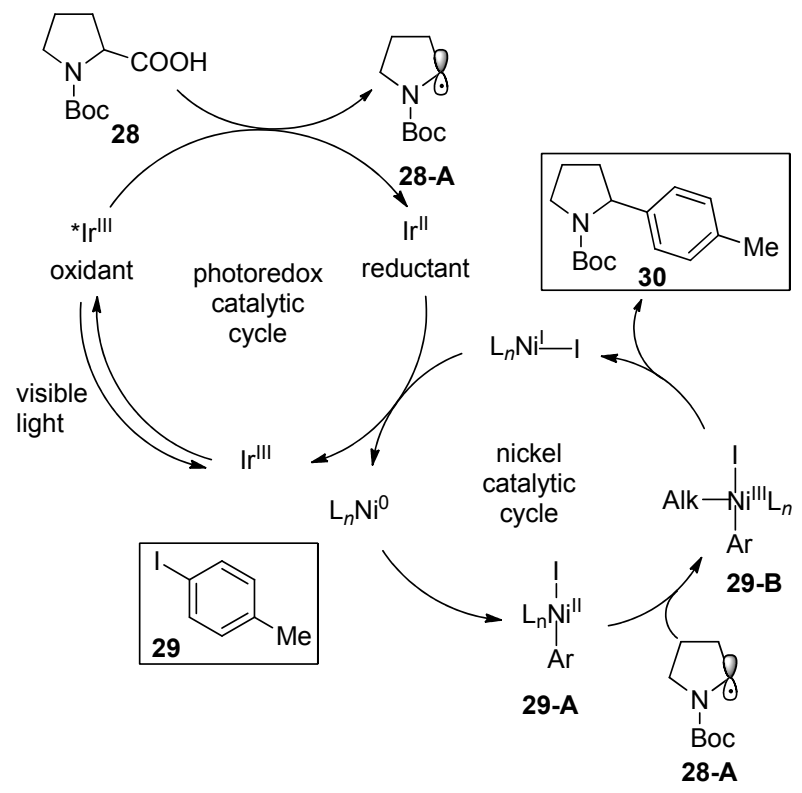

Scheme 11 镍和光催化剂协同催化的 $\mathrm{C}\left(\mathrm{sp}^{3}\right)-\mathrm{C}\left(\mathrm{sp}^{2}\right)$ 烷基羧 酸与芳基卤化物的脱羧偶联机理

Scheme 11 Proposed mechanism for the dual nickel photoredox $\mathrm{C}\left(\mathrm{sp}^{3}\right)-\mathrm{C}\left(\mathrm{sp}^{2}\right)$ cross coupling between aryl halides and carboxylic acids

利用光催化下的这一 $\mathrm{Ni}(0) \rightarrow \mathrm{Ni}(\mathrm{II}) \rightarrow \mathrm{Ni}(\mathrm{III}) \rightarrow \mathrm{Ni}(\mathrm{I})$ 机制, MacMillan 小组 ${ }^{[20]}$ 于 2015 年报道了利用醇和芳基 卤化物在镍和光催化剂的共同作用下偶联为芳基醚的 反应. 该反应直接利用处于氧化态的光敏剂将不易发生 还原消除的 $\mathrm{Ni}(\mathrm{II})$ 物种氧化为 $\mathrm{Ni}(\mathrm{III})$ 物种, 从而促进还 原消除，实现了 $\mathrm{C}\left(\mathrm{sp}^{2}\right)-\mathrm{O}$ 偶联反应(Scheme 12).

除了 $\mathrm{C}\left(\mathrm{sp}^{3}\right)-\mathrm{C}\left(\mathrm{sp}^{2}\right)$ 偶联反应，这一机制还被应用 于 $\mathrm{C}-\mathrm{P}$ 键的构建中. 2015 年, 肖文精课题组 ${ }^{[21]}$ 报道了 镍和光催化剂协同催化的二芳基氧磷与芳基碘化物的 偶联反应. 该反应具有广泛的底物范围, 可以兼容各种 官能团，产率最高可达 91\% (Eq. 11).

\section{3 可见光催化促进的转金属化反应}

转金属化反应是亲核底物与金属中心的成键作用,

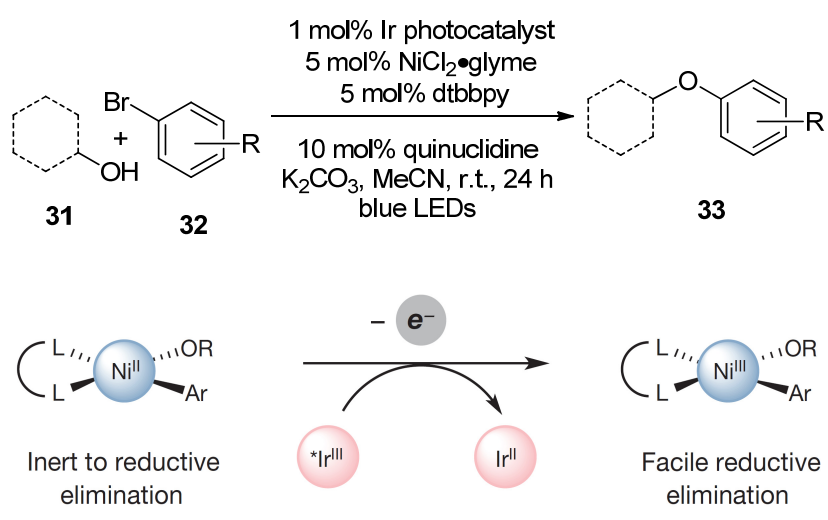

Scheme 12 镍和光催化剂协同催化的 $\mathrm{C}\left(\mathrm{sp}^{2}\right)-\mathrm{O}$ 醇与芳基卤 化物的偶联反应

Scheme 12 Dual nickel photoredox $\mathrm{C}\left(\mathrm{sp}^{2}\right)-\mathrm{O}$ cross coupling between aryl halides and alcohols

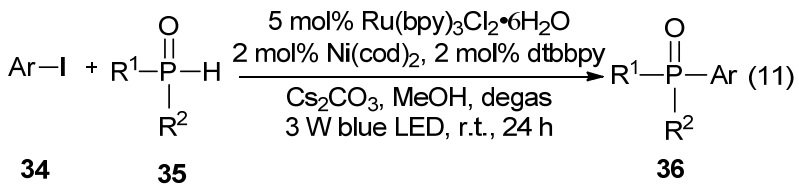

形式上金属价态没有变化, 但是, 光催化的介入也可以 促进转金属化反应. 其一般的机理可能是：首先，亲核 底物通过单电子转移的机制在光催化的条件下被氧化 为自由基，然后加成到金属上形成配合物，这一配合物 中间体再被光催化还原为低价态. 基于这一原理, 2014 年 Molander 课题组 ${ }^{[22]}$ 实现了苄基三氟硼酸钾与芳基溴 化物在光催化剂和镍催化剂协同作用下的偶联反应，该 反应经由光促进的单电子转移机制完成转金属化反应, 从而克服了传统转金属化的高能垒(Eq. 12).

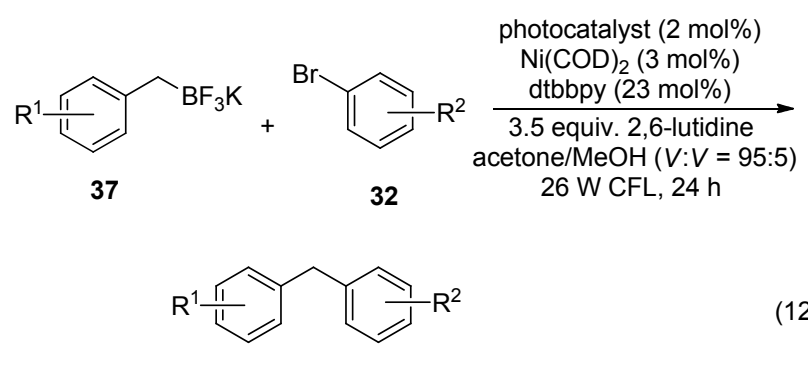

38

up to $97 \%$ yield

对于具体的反应机理，作者认为首先芳基卤化物与 $\mathrm{Ni}(0)$ 发生氧化加成生产 $\mathrm{Ni}(\mathrm{II})$ 中间体，同时，茮基三氟 嗍酸钾在光催化条件下脱三氟硼酸基产生烷基自由基, 然后该自由基加成到 $\mathrm{Ni}(\mathrm{II})$ 中间体上生成 $\mathrm{Ni}$ (III)中间体. 接着, $\mathrm{Ni}(\mathrm{III})$ 中间体再发生还原消除生成 $\mathrm{Ni}(\mathrm{I})$ 中间体, 最后 $\mathrm{Ni}(\mathrm{I})$ 再被处于低价态的光敏剂还原为 $\mathrm{Ni}(0)$ (Scheme 13). 


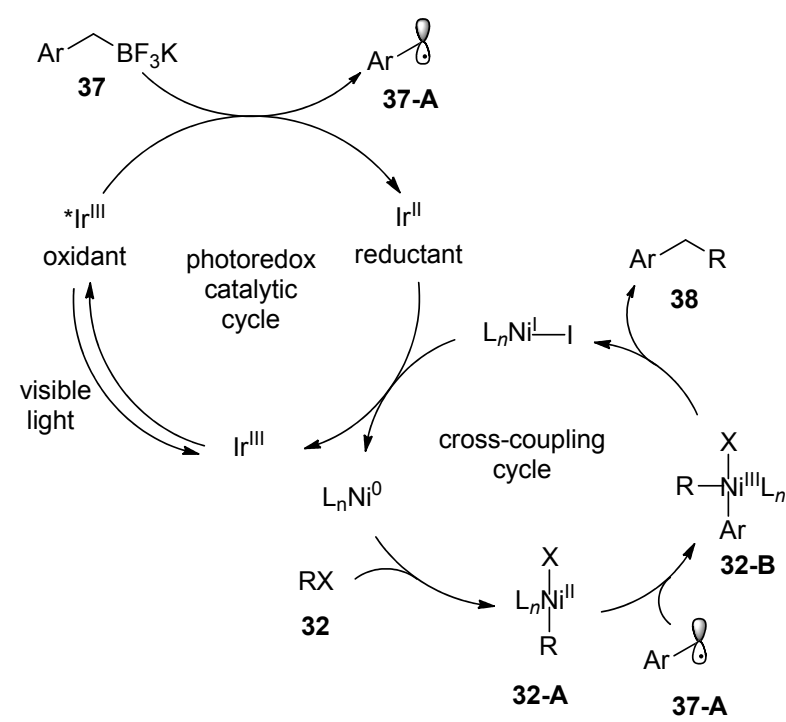

Scheme 13 镍和光催化剂协同催化的苄基三氟硼酸钾与芳 基溴化物的偶联反应机理

Scheme 13 Proposed mechanism for dual nickel photo redox cross coupling between diarylphosphine oxides and aryl iodides

\section{4 结论}

本综述阐述了可见光催化促进的金属有机基元反 应, 包括氧化加成、还原消除以及转金属化反应等. 这 类反应的特点是利用单电子转移的方式实现金属的氧 化还原，从而实现传统金属有机中较为困难的反应. 目 前这一策略的研究主要集中在钯、金、镍等金属催化的 反应上, 未来的研究有望进一步拓展到其它金属, 特别 是廉价金属催化的反应. 另外, 这些反应的其中一个偶 联组分一一自由基源的类型太少, 大多数采用的是芳基 重氮盐和二芳基高碘盐. 我们相信, 在不久的将来, 更 多的偶联组分可被应用于该反应.

\section{References}

[1] (a) Johansson Seechurn, C. C. C.; Kitching, M. O.; Colacot, T. J.; Snieckus, V. Angew. Chem., Int. Ed. 2012, 51, 5062.

(b) Hartwig, J. F. Organotransition Metal Chemistry: From Bonding to Catalysis, University Science Books, Herndon, VA, 2010.

[2] For selected reviews on photoredox catalysis: (a) Xuan, J.; Xiao, W. J. Angew. Chem., Int. Ed. 2012, 51, 6828.

(b) Prier, C. K.; Rankic, D. A.; MacMillan, D. W. Chem. Rev. 2013, 113,5322 .

(c) Schultz, D. M.; Yoon, T. P. Science 2014, 343, 1239176

(d) Angnes, R. A.; Li, Z.; Correia, C. R. D.; Hammond, G. B. Org. Biomol. Chem. 2015, 13, 9152.

(e) Xuan, J.; Zhang, Z. G.; Xiao, W. J. Angew. Chem., Int. Ed. 2015, 54,15632 .

(f) Luo, J.; Zhang, J. ACS Catal. 2016, 6, 873.

(g) Chen, J. R.; Hu, X. Q.; Lu, L. Q.; Xiao, W. J. Chem. Soc. Rev. 2016, 45, 2044.

(h) Lang, X.; Zhao, J.; Chen X., Chem. Soc. Rev. 2016, 45, 3026.

(i) Levin, M. D.; Kim, S.; Toste, F. D. ACS Cent. Sci. 2016, 2, 293.

(j) Skubi, K. L.; Blum, T. R.; Yoon, T. P. Chem. Rev. 2016, 116,
10035 .

(d) Hopkinson, M. N.; Tlahuext-Aca, A.; Glorius, F. Acc. Chem. Res. 2016, 49, 2261

(k) Tellis, J. C.; Kelly, C. B.; Primer, D. N.; Jouffroy, M.; Patel, N. R.; Molander, G. A. Acc. Chem. Res. 2016, 49, 1429.

(1) Gui, Y.-Y.; Sun, L.; Lu, Z.-P.; Yu, D.-G. Org. Chem. Front. 2016, 3,522 .

(m) Fabry, D.-C.; Rueping, M. Acc. Chem. Res. 2016, 49, 1969.

(n) Zhang, M.; Zhu, C.; Ye, L.-W. Synthesis 2017, 1150.

(o) Zuo, X.; Wu, W.-L.; Su, W.-P. Acta Chim. Sinica 2015, 73, 1298 (in Chinese).

(左璇，吴文亮，苏伟平，化学学报，2015, 73, 1298.)

(p) Guan, B.-C.; Xu, X.-L.; Wang, H.; Li, X.-N. Chin. J. Org. Chem. 2016, 36, 1564 (in Chinese).

(关保川, 许孝良, 王红, 李小年, 有机化学, 2016, 36, 1564.)

(q) Tan, F.; Xiao, W.-J. Acta Chim. Sinica 2015, 73, 85 (in Chinese).

(谭芬, 肖文精, 化学学报, 2015, 73, 85.)

(r) Roh, G.-B.; Iqbal N.; Cho. E. J. Chin. J. Chem. 2016, 34, 459.

[3] Halpern, J. Acc. Chem. Res. 1970, 3, 386.

[4] Hill, R. H.; Puddephatt, R. J. J. Am. Chem. Soc. 1985, 107, 1218.

[5] (a) Johnson, A.; Puddephatt, R. J. J. Chem. Soc., Dalton Trans. 1976, 1360.

(b) Winston, M. S.; Wolf, W. J.; Toste, F. D. J. Am. Chem. Soc. 2014, 136, 7777.

[6] (a) Kalyani, D.; McMurtrey, K. B.; Neufeldt, S. R.; Sanford, M. S. J. Am. Chem. Soc. 2011, 133, 18566.

(b) Neufeldt, S. R.; Sanford, M. S. Adv. Synth. Catal. 2012, 354, 3517.

[7] Sahoo, B.; Hopkinson, M. N.; Glorius, F. J. Am. Chem. Soc. 2013, 135,5505 .

[8] Hopkinson, M. N.; Sahoo, B.; Glorius, F. Adv. Synth. Catal. 2014 356, 2794.

[9] Xia, Z.; Khaled, O.; Mouriès-Mansuy, V.; Ollivier, C.; Fensterbank, L. J. Org. Chem. 2016, 81, 7182 .

[10] Shu, X.-Z.; Zhang, M.; He, Y.; Frei, H.; Toste, F. D. J. Am. Chem. Soc. 2014, 136, 5844.

[11] Um, J.; Yun, H.; Shin, S. Org. Lett. 2016, 18, 484

[12] Tlahuext-Aca, A.; Hopkinson, M. N.; Garza-Sanchez, R. A.; Glorius, F. Chem.-Eur. J. 2016, 22, 5909.

[13] Alcaide, B.; Almendros, P.; Busto, E.; Luna, A. Adv. Synth. Catal. 2016, 358,1526

[14] He, Y.; Wu, H.; Toste, F. D. Chem. Sci. 2015, 6, 1194.

[15] Tlahuext-Aca, A.; Hopkinson, M. N.; Sahoo, B.; Glorius, F. Chem. Sci. 2016, 7, 89

[16] Kim, S.; Rojas-Martin, J.; Toste, F. D. Chem. Sci. 2016, 7, 85.

[17] Cornilleau, T.; Hermange, P.; Fouquet, E. Chem. Commun. 2016 $52,10040$.

[18] Gauchot, V.; Lee, A.-L. Chem. Commun. 2016, 52, 10163

[19] (a) Zuo, Z.; Ahneman, D. T.; Chu, L.; Terrett, J. A.; Doyle, A. G.; MacMillan, D. W. C. Science 2014, 345, 437.

(b) Le, C. C.; MacMillan, D. W. C. J. Am. Chem. Soc. 2015, 137, 11938.

(c) Noble, A.; McCarver, S. J.; MacMillan, D. W. C. J. Am. Chem. Soc. 2015, 137, 624 .

[20] (a) Terrett, J. A.; Cuthbertson, J. D.; Shurtleff, V. W.; MacMillan, D. W. C. Nature 2015, 524, 330

(b) Chu, L.; Lipshultz, J. M.; MacMillan, D. W. C. Angew. Chem., Int. Ed. 2015, 54, 7929.

[21] Xuan, J.; Zeng, T.; Chen, J.; Lu, L.; Xiao, W. Chem.-Eur. J. 2015 , $21,4962$.

[22] (a) Tellis, J. C.; Primer, D. N.; Molander, G. A. Science 2014, 345, 433.

(b) El Khatib, M.; Serafim, R. A. M.; Molander, G. A. Angew. Chem., Int. Ed. 2016, 55, 254.

(c) Primer, D. N.; Karakaya, I.; Tellis, J. C.; Molander, G. A. J. Am. Chem. Soc. 2015, 137, 2195. 